\title{
An Introduction to Energy Demand Challenges in Europe
}

\author{
Frances Faby and Gary Goggins
}

\begin{abstract}
This opening chapter calls for greater attention to energy demand challenges in Europe. It argues that many obstacles and opportunities in achieving the so-called energy transition are social and cultural in nature and require interdisciplinary solutions that go beyond efficiency approaches. We provide an overview of the ENERGISE project that aims to achieve greater understanding of the social and cultural influences on household energy use, and to develop appropriate responses and recommendations for policy-makers, practitioners and future academic research. The chapter concludes with a brief summary of the structure of this book, including an introductory overview of how energy demand challenges are understood, and how this relates to the types of solutions that are proposed in each of the ten European countries studied.
\end{abstract}

F. Fahy $(\square) \cdot$ G. Goggins

School of Geography and Archaeology and Ryan Institute,

National University of Ireland Galway, Galway, Ireland

e-mail: frances.fahy@nuigalway.ie

G. Goggins

e-mail: gary.goggins@nuigalway.ie

(C) The Author(s) 2019

F. Fahy et al. (eds.), Energy Demand Challenges in Europe, https://doi.org/10.1007/978-3-030-20339-9_1 
Keywords Energy policy • Europe • Horizon 2020 • Interdisciplinary • ENERGISE

\section{Exploring Energy Demand Challenges in Europe}

Significant challenges lie ahead regarding Europe's transition towards a decarbonised energy system that meets the economic and social needs of its citizens. Heretofore, scientific research and public policy in the field of household energy use has primarily focused on promoting energy efficiency through changes in technology and individual behaviour (Labanca and Bertoldi 2018). However, these approaches have been ineffective in bringing about the aggregated reductions in carbon emissions that are necessary to meet climate targets. They may even be counterintuitive if they reinforce unsustainable routines and habits that engage energyrelated services (Shove 2018; Hargreaves et al. 2018).

The performance of more efficient technologies is often dependent on how they are used by householders, if at all. Moreover, short-term efficiency gains may be wiped out by increasing overall consumption in order to reach newly perceived levels of comfort, convenience and standards. This increase in consumption manifests, for example, through social pressure to consume in line with the latest 'trends' for bigger houses, bigger cars, the latest technologies and appliances, and so forth. At the same time, many households experience energy poverty and are unable to meet their energy needs, such as providing adequate heat for their homes. Addressing these concerns requires multi-dimensional approaches and highlights the crucial role of consumption in multi-scalar decarbonisation efforts across Europe, an issue requiring much greater attention from scientists and policy-makers than before.

This calls for a broadening of discussions around energy use to include such fundamental and deep-rooted questions as 'How much of what is enough?' Of course, finding consensus on a clearly subjective issue is never going to be easy, or indeed possible. But, by framing the energy challenge in this way, we are compelled to be reflexive in considering how collective conventions around energy use evolve, and how can they be better aligned with sustainable lifestyles. Opening up these increasingly pertinent discussions to wider debate also implies a greater emphasis on citizen engagement to respond to related social and cultural 
challenges through more participatory processes. Undertaking these democratic exercises can help us to better comprehend societal norms and routines that greatly determine our patterns of energy use as well as our ability to change those patterns. By understanding and accounting for particular householder needs and other contextual conditions of consumption, policy-makers and practitioners can tailor sustainable energy responses accordingly.

This book gathers together contributions from prominent social scientists researching in the energy field all across Europe. While the authors hail from diverse disciplinary backgrounds, they all recognise that cultural- and systemic change is a key ingredient in successful energy transitions. The book offers unique and often fascinating insights into the socio-material similarities and differences in energy policies, energy infrastructures and energy demands across 10 European countries. The collection provides invaluable accounts of the diverse contexts within which individuals, households and communities engage in everyday energy-related practices, and the policies and practices that underpin ongoing efforts towards sustainable transformation.

\section{The ENERGise Project as Context}

This book is a key output from the ENERGISE project. ENERGISE is an innovative pan-European research initiative to achieve a greater scientific understanding of the social and cultural influences on energy consumption. Funded under the EU Horizon 2020 programme for three years (2016-2019), ENERGISE develops, tests and assesses options for a bottom-up transformation of energy use in households and communities across Europe.

Energy use can be fruitfully understood as engaging in a set of practices that incorporate elements of meaning, skills and material conditions and that are embedded in wider social, political and institutional contexts (Shove et al. 2012). This practice-oriented perspective is central to the ENERGISE project for a number of reasons. First, a practice perspective departs from individualistic views of energy choices and behaviour that have unduly limited past research on energy demand and its transformation. Instead, the focus shifts towards an understanding of energy behaviour as collectively shared and culturally mediated. Second, an explicit focus on energy-related practices promotes cutting-edge social-scientific 
and interdisciplinary energy research that covers both social and material aspects of energy use.

In particular, ENERGISE investigates the energy-related practices of households and communities and their impacts on society and the environment. This is achieved through a living lab approach, where researchers work with households in a real-world setting and in the context within which their energy use takes place. ENERGISE Living Labs (ELLs) build on interactions between various stakeholders as well as lessons drawn from previous initiatives aimed at reducing household energy use, or what we term sustainable energy consumption initiatives (SECIs). In ENERGISE, SECIs are defined as activities that deal with reducing energy-related $\mathrm{CO}_{2}$ emissions from households, and enables active participation from households. This can either be in terms of (1) reducing the actual energy consumption, (2) reducing the emissions intensity of energy consumption (e.g. by substituting fossil fuels with renewable energy sources). Sustainable energy initiatives are considered to be socio-technical because they attempt to change the material arrangements and the cultures, norms and conventions that determine collective energy use and related impacts. Examples of good practice SECIs that have informed the design and implementation of ELLs are provided for each of the central chapters in the book. The results of the ELLs themselves are presented elsewhere.

\section{REMiT OF THE BOOK}

The following chapter will introduce the concept of 'problem framings' related to the way energy consumption challenges are understood and the impact for the type of solutions that emerge. Based on the review of SECIs already carried out across Europe as part of the ENERGISE project, it is evident that many different perspectives on sustainable energy use exist and problems are addressed in a number of different ways.

Energy use is typically interpreted as a matter of individual choices and preferences that can be changed independently of the context within which consumption occurs, and often through technological optimisation or incremental behaviour change. This approach, however, often fails to translate into significant changes in energy consumption patterns and energy demands. In contrast, other more integrated approaches treat energy consumption challenges as a matter of wider societal, cultural and 
institutional dynamics, which suggest that changes in energy demand will come about only if the entire range of elements that underpin energy use are considered. These alternative approaches set out to obtain quite different results; some suggest qualitative changes in habits and routinised activities, while others set sufficiency-based targets that seek absolute reductions in energy use (or increases for those in energy poverty) and set minimum and maximum limits to consumption. Illustrated with examples, Chapter 2 will introduce how these different approaches or problem framings can be characterised, and what the different framings mean for the objectives, methods and assumptions made in sustainable energy consumption initiatives.

The central chapters in this collection provide insights into trends in energy transitions and what this means for the future of energy demand in Europe. The inclusion of ten chapters, each focusing on a different European country, offers a broad yet accessible overview of the diverse energy systems currently in place, albeit in a rapidly changing context. What is particularly evident is that no one system is free from contestation over how to best meet present and future energy needs. Every country is unique in the energy-related challenges they face, with each having different institutional structures and capacity to deal with problems and to capitalise on opportunities. At the same time, all countries share common concerns around issues such as energy security, affordability and sustainability, and all have signed up to international climate change agreements that call for dramatic reductions in carbon emissions.

Another interesting observation is how energy policy is evolving across the countries under study. Emerging trends include the changing role of citizens in the energy transition, more generally portrayed as shifting from 'passive' to 'active' consumers, but manifesting in a number of different forms from 'prosumers' to 'energy communities'. Householders are also envisaged to play a central role as the shift toward 'smart' grid solutions and systems intensifies. Driving these changes requires robust policy responses and the engagement of actors at all levels of society including government bodies, researchers, businesses, NGOs and community groups.

A recent phenomenon that facilitates greater engagement in decision-making has been a political shift across much of Europe toward multilevel governance. This has facilitated greater societal engagement with the sustainable energy agenda and provided new avenues for non-state actors to play a significant role in developing responses. 
The expansion of the spatial and political boundaries in which responses occur is reflected in the increasing range of actors, sites, configurations and mechanisms through which sustainable energy is being addressed, as demonstrated in the case studies provided in this book.

The final chapter within this collection reflects on the key strands emerging from the material presented and considers individual and collective opportunities for sustainable energy transitions. In comparing and contrasting energy-related problem framings and social, material and institutional make-up across Europe, the concluding chapter unpacks the energy challenges facing Europe. It clearly shows that policies for energy demand reduction have to carefully consider and address the differences in cultural, material and institutional constitutions of energy demand and energy systems, locally, regionally, nationally and cross-nationally.

\section{REFERENCES}

Hargreaves, T., Wilson, C., \& Hauxwell-Baldwin, R. (2018). Learning to live in a smart home. Building Research \& Information, 46(1), 127-139.

Labanca, N., \& Bertoldi, P. (2018). Beyond energy efficiency and individual behaviours: Policy insights from social practice theories. Energy Policy, 115, 494-502.

Shove, E. (2018). What is wrong with energy efficiency? Building Research \& Information, 46(7), 779-789.

Shove, E., Pantzar, M., \& Watson, M. (2012). The dynamics of social practice: Everyday life and how it changes. Los Angeles: Sage. 
Open Access This chapter is licensed under the terms of the Creative Commons Attribution 4.0 International License (http://creativecommons.org/licenses/ by $/ 4.0 /$ ), which permits use, sharing, adaptation, distribution and reproduction in any medium or format, as long as you give appropriate credit to the original author(s) and the source, provide a link to the Creative Commons license and indicate if changes were made.

The images or other third party material in this chapter are included in the chapter's Creative Commons license, unless indicated otherwise in a credit line to the material. If material is not included in the chapter's Creative Commons license and your intended use is not permitted by statutory regulation or exceeds the permitted use, you will need to obtain permission directly from the copyright holder.

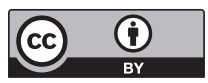

